Research paper

\title{
Self-reported disability and quality of life in an online Australian community sample with suicidal thoughts
}

\author{
Bregje A.J. van Spijker ${ }^{\mathrm{a}}$, Philip J. Batterham ${ }^{\mathrm{a}}$, Alison L. Calear ${ }^{\mathrm{a}, *}$, Quincy J.J. Wong ${ }^{\mathrm{b}, \mathrm{c}}$, \\ Aliza Werner-Seidler ${ }^{\mathrm{b}}$, Helen Christensen ${ }^{\mathrm{b}}$ \\ ${ }^{\text {a }}$ Centre for Mental Health Research, Research School of Population Health, The Australian National University, Canberra, Australia \\ ${ }^{\mathrm{b}}$ Black Dog Institute, University of New South Wales, Sydney, NSW, Australia \\ ${ }^{\mathrm{c}}$ School of Social Sciences and Psychology, Western Sydney University, Sydney, NSW, Australia
}

\section{A R T I C L E I N F O}

\section{Keywords:}

Suicide

Disability

Physical health

Mental health

Quality of life

\begin{abstract}
A B S T R A C T
Background: Limited research has been conducted to quantify the level of disability and health related quality of life (HRQoL) associated with suicidal thoughts and behaviour. The aims of the current study were to (1) describe levels of disability and HRQoL in an Australian sample of adults with suicidal ideation, (2) examine the effect of zero, one, or more than one previous suicide attempt on disability and HRQoL, and (3) describe the demographic and clinical characteristics associated with disability and HRQoL.

Method: Data for the current study is drawn from the baseline assessment of the Healthy Thinking trial that evaluated the effectiveness of an online self-help program for suicidal thoughts in an Australian adult community sample $(n=418)$. Measures of disability, quality of life, clinical symptoms, suicidality and demographic variables were included.

Results: The mean disability score for the overall sample was $19.2(S D==8.9)$, while the mean perceived physical health score was $40.7(S D==6.5)$ and the mean perceived mental health score was $36.1(S D==5.9)$. Higher levels of disability and lower levels of perceived physical health were associated with multiple suicide attempts compared to one or no history of suicide attempt.

Limitations: Limitations included the limited generalisability of results, the use of self-report to assess suicide attempts, and the use of cross-sectional data.

Conclusion: Suicidal thoughts and behaviours are associated with high levels disability and low levels of perceived physical and mental health. Early intervention programs are needed to reduce the high levels of burden associated with suicidality.
\end{abstract}

\section{Introduction}

While self-harm and suicide deaths are recognised as leading causes of disease burden worldwide (G.B.D. DALYs and HALE Collaborators, 2017; Karimkhani et al., 2016; Naghavi, 2019), there is limited evidence for the specific effects of suicidal ideation on Health Related Quality of Life (HRQoL). Furthermore, the factors associated with decrements in HRQoL for people experiencing suicidal thoughts are poorly understood. Terminology used in quality of life research is sometimes imprecise, requiring clarity of definitions for key concepts (Karimi and Brazier, 2016; Post, 2014). The current study defines the term Health Related Quality of Life (HRQoL) as a self-assessed ability to function in the physical, mental and social domains of life. Disability in this study is defined as "a difficulty in functioning at the body, person, or societal levels, in one or more life domains, as experienced by an individual with a health condition in interaction with contextual factors" (Leonardi et al., 2006). While the concepts of HRQoL and disability are closely related, they reflect distinct constructs, with disability taking into account contextual or environmental factors (Leonardi et al., 2010). It is proposed that suicidal thinking may reduce HRQoL through reduced perceived functioning in physical, mental and/ or social domains, and the consequent limitations in functioning may lead to disability particularly among people with chronic suicidal thoughts (van Spijker et al., 2011). The correspondence between HRQoL and disability is often imperfect (Albrecht and Devlieger, 1999), so it is necessary to independently evaluate the effects of suicidal ideation on both constructs.

Only a few studies have examined the association between

\footnotetext{
* Corresponding author.

E-mail address: alison.calear@anu.edu.au (A.L. Calear).
} 
Table 1

Sample characteristics.

\begin{tabular}{|c|c|c|c|c|c|}
\hline & Full sample $(N=418)$ & Ideation only $(n=191)$ & Single attempt $(n=89)$ & Multiple attempts $(n=138)$ & \\
\hline Age $m(S D)$ & $40.64(11.94)$ & 41.77 (12.67) & 39.93 (11.39) & $39.52(11.16)$ & \\
\hline \multicolumn{6}{|l|}{ Gender $^{\mathrm{a}}$} \\
\hline Male $n(\%)$ & $93(22.2)$ & $53(27.7)$ & 15 (16.9) & $25(18.1)$ & \\
\hline Female $n(\%)$ & $323(77.3)$ & $138(72.3)$ & $74(83.1)$ & $111(80.4)$ & \\
\hline \multicolumn{6}{|l|}{ Relationship status } \\
\hline Married/de facto $n(\%)$ & $160(38.3)$ & $82(42.9)$ & $31(34.8)$ & $47(34.1)$ & \\
\hline Separated/divorced/widowed $n(\%)$ & $72(17.2)$ & $30(15.7)$ & $16(18.0)$ & $26(18.8)$ & \\
\hline Single $n(\%)$ & $186(44.5)$ & $79(41.4)$ & $42(47.2)$ & $65(47.1)$ & \\
\hline \multicolumn{6}{|l|}{ Education } \\
\hline No post-school education & $69(16.5)$ & $30(15.7)$ & $13(14.6)$ & $26(18.8)$ & \\
\hline Trade or other certificate & $103(24.6)$ & $46(24.1)$ & $22(24.7)$ & $35(25.4)$ & \\
\hline Associate degree or higher & $246(58.9)$ & $115(60.2)$ & $54(60.7)$ & $77(55.8)$ & \\
\hline Employment status & & & & & * \\
\hline Employed fulltime $n(\%)$ & $155(37.1)$ & $89(46.6)$ & $34(28.2)$ & $32(23.2)$ & \\
\hline Employed part-time & $93(22.2)$ & $40(20.9)$ & $26(29.2)$ & $27(19.6)$ & \\
\hline Unemployed $n(\%)$ & $66(15.8)$ & $27(14.1)$ & $13(14.6)$ & $26(18.8)$ & \\
\hline Not in labour force $n(\%)$ & $104(24.9)$ & $35(18.3)$ & $16(18.0)$ & $53(38.4)$ & \\
\hline \multicolumn{6}{|l|}{ Region $^{\mathrm{a}}$} \\
\hline Metropolitan $n(\%)$ & $253(60.5)$ & $125(65.4)$ & $49(55.1)$ & $79(57.2)$ & \\
\hline Regional $n(\%)$ & $107(25.6)$ & $43(22.5)$ & $29(32.6)$ & $35(25.4)$ & \\
\hline Rural/Remote $n$ (\%) & $56(13.4)$ & $22(11.5)$ & $11(12.4)$ & $23(16.7)$ & \\
\hline \multicolumn{6}{|l|}{ Clinical characteristics } \\
\hline Depressive symptoms & $40.26(9.62)$ & $39.06(8.88)$ & 39.99 (9.69) & 42.09 (10.33) & * \\
\hline Anxiety symptoms & $13.27(5.07)$ & $12.90(5.11)$ & $12.78(5.40)$ & $14.10(4.70)$ & \\
\hline Insomnia & $15.34(5.96)$ & $14.10(5.81)$ & $16.04(5.84)$ & $16.61(5.93)$ & * \\
\hline Alcohol Consumption & $3.07(2.95)$ & $2.93(2.85)$ & $3.38(3.00)$ & $3.07(3.06)$ & \\
\hline C-SSRS ideation severity & $15.26(3.15)$ & $14.35(3.17)$ & $15.61(2.86)$ & $16.29(2.95)$ & * \\
\hline \multicolumn{6}{|l|}{ Lifetime suicide attempt } \\
\hline Ideation only & $191(45.7)$ & - & - & - & \\
\hline Single attempt & $89(21.3)$ & - & - & - & \\
\hline Multiple attempts & $138(33.0)$ & - & - & - & \\
\hline Disability (WHODAS-II) & $19.20(8.91)$ & $17.14(8.40)$ & $18.93(8.78)$ & 22.24 (8.89) & * \\
\hline SF-12 PCS & $40.67(6.49)$ & $41.77(6.51)$ & $41.05(6.29)$ & $38.92(6.25)$ & * \\
\hline SF-12 MCS & $36.05(5.94)$ & $36.13(5.46)$ & $36.52(6.27)$ & $35.65(6.35)$ & \\
\hline
\end{tabular}

Note. C-SSRS = Columbia-Suicide Severity Rating Scale; WHODAS-II = World Health Organization Disability Assessment Schedule-II; SF-12 = Short Form-12; PCS = Physical Component Scale; MCS = Mental Component Scale.

aissing: $n=2$ for gender and region.

${ }^{*} p<0.05$ based on $\chi^{2}$ tests (categorical variables) or $F$ tests (continuous variables) comparing ideation only vs. single attempt vs. multiple attempts.

suicidality and disability. One of these studies calculated disability weights and showed that suicidal thoughts are comparable in terms of associated disability to severe asthma, moderate heart failure, alcohol dependence, and cocaine dependence (van Spijker et al., 2011). Psychological distress associated with suicide attempts was found to be as disabling as heroin dependence and the early stages of Parkinson's disease. Findings from this study also suggested that the disability for suicide attempts may largely be attributable to the psychological distress involved, as the disability weight for physical injury following a suicide attempt (estimated by Hoeymans and Schoemaker (2010)) was found to be much lower. A recent review into functional disability and suicidal behaviour in middle-aged and older adults reported that the majority of the 45 included studies found a significant association, but concluded that more research is needed to clarify the specifics of these associations (Lutz and Fiske, 2017).

There have also been few studies assessing the impact of suicidality on HRQoL. Fairweather-Schmidt et al. (2016) found markedly lower mental HRQoL in community-dwelling adults with suicidal thoughts compared to those with no suicidal thoughts. In contrast, physical HRQoL was slightly higher in the group with suicidal thoughts compared to people with no suicidal thoughts. An investigation of longitudinal trajectories showed that mental HRQoL did not change over time, while physical HRQoL deteriorated marginally (FairweatherSchmidt et al., 2016). Similarly, Sinclair et al. (2010) assessed quality of life in a group of clinical self-harm patients and also reported worse quality of life compared with local population norms (Sinclair et al., 2010).

Research into the disability and HRQoL associated with suicidal thoughts may allow comparisons with other health problems. This has been illustrated by Cook and Harman (2008) who compared chronic medical conditions and mental health disorders and showed the latter to be responsible for significantly greater impairment. Such information also provides a basis for policy makers and research funders, which is of particular importance given limited funding for suicide prevention (Christensen et al., 2015). In addition, HRQoL concepts can assist in quantifying potential benefits of reducing suicidal ideation in the community (e.g., what level of disability can be averted), adding to the decision making toolkit.

No previous study has examined factors associated with disability or HRQoL among individuals with suicidal ideation, and little research has examined whether individuals reporting a suicide attempt have greater disability or poorer HRQoL compared to those who experience ideation without attempt. This study uses data from a randomised controlled trial that recruited adults with suicidal thoughts from the Australian general population and aims to (1) describe levels of disability and HRQoL in this sample at baseline, (2) examine whether participants who report one or multiple previous suicide attempts experience significantly higher disability and lower HRQoL compared with participants who only report suicidal thoughts, and (3) describe the demographic and clinical characteristics associated with disability and HRQoL among individuals experiencing suicidal thoughts. It was hypothesized levels of disability would be high and HRQoL would be poor and in this sample, with additional decrements attributable to one or multiple attempts. 


\section{Methods}

\subsection{Overview}

This study is a secondary analysis of the Healthy Thinking trial, which is a randomised controlled trial (RCT) that investigated the effectiveness of an online self-help program for suicidal thoughts in an Australian community sample. The rationale, methods, and primary analyses have been published elsewhere (van Spijker et al., 2015, 2018), but key elements of the methods relevant to the current analyses are described below.

\subsection{Participants}

Participants were recruited between November 2013 and December 2015 via various online media (e.g., websites, social networking websites, and advertising on search engines). Interested respondents were screened for eligibility online via self-report on the following criteria: (1) aged between 18 and 65 years, (2) have a valid email address, (3) have a reliable internet connection, (4) located in Australia, (5) fluent in English, (6) no history of a diagnosed psychotic disorder, (7) current suicidal thoughts, and (8) no recent (i.e., past-month) suicide attempt. A total of 418 participants who were deemed eligible completed enrolment and were included. The current study uses data from the baseline assessment of the trial, which was conducted online via selfreport. Demographics and clinical characteristics for the full sample and subsamples of participants with ideation only, a single previous suicide attempt and multiple previous suicide attempts are reported in Table 1.

\subsection{Measures}

\subsubsection{Disability}

The World Health Organization Disability Assessment Schedule-II (WHODAS-II) measures disability at a population level or in clinical practice. It captures the level of functioning in six domains of life: cognition, mobility, self-care, getting along with others, life activities, and participation in society. The short version of the WHODAS-II, which consists of 12 self-report items that relate to difficulties experienced in these six domains during the previous 30 days, was used in this study. The items are scored on a 0 ("none") to 4 ("extreme/cannot do") scale. Total scores were computed with a simple sum scoring method and can range from 0 (no disability) to 48 (complete disability) (Andrews et al., 2009; Üstün et al., 2010). The 12-item version of the WHODAS-II has been shown to be a reliable and valid measure of global disability in the Australian context (Andrews et al., 2009). In addition, the WHODAS-II includes five items that assess: overall health (endorsed on a 5-point scale ranging from "very bad" to "very good"), interference with daily life (endorsed on a 5-point scale ranging from "not at all" to "extremely"), number of days difficulties were present, number of days respondents were totally unable to carry out their usual activities or work, and number of days respondents had to reduce their usual activities or work. Internal consistency was good $(\alpha=0.89)$ in the present sample.

\subsubsection{Health-related quality of life}

The Short Form-12 (SF-12) is a generic 12-item measure of perceived HRQoL that provides two summary measures: the Physical Component Scale (PCS) and the Mental Component Scale (MCS). It has been shown to have similar performance characteristics to the longer, 36 item version (Jenkinson et al., 1997; Ware et al., 1996). Responses were binary (yes/no) or on a Likert scale. Higher scores indicate better physical or mental quality of life. These summary scores are transformed to the norm-based scoring so that means of 50 and standard deviations of 10 on each Component Scale are achieved (Ware et al., 1995). The PCS had good internal consistency $(\alpha=0.75)$, while the MCS had adequate internal consistency $(\alpha=0.64)$ in the present sample.

\subsubsection{Predictor variables}

Demographic predictor variables explored include age, gender, relationship status, education, employment, and region (metropolitan, regional, rural/remote). Clinical predictor variables were depressive symptoms [assessed with the Centre for Epidemiological Studies, CES-D (Sawyer Radloff, 1977), $\alpha=0.87$ ], anxiety symptoms [assessed with the Generalised Anxiety Disorder-7, GAD-7 (Spitzer et al., 2006), $\alpha=0.85$ ], insomnia [assessed with the Insomnia Severity Index, ISI (Morin et al., 2011), $\alpha=0.80$ ], alcohol consumption [assessed with the Alcohol Use Disorders Identification Test Consumption, AUDIT-C (Bush et al., 1998), $\alpha=0.75$ ], severity of suicidal ideation [assessed with the Columbia-Suicide Severity Rating Scale, C-SSRS (Posner et al., 2011), $\alpha=0.62$ ], and suicide attempt history (assessed with a single question).

\subsection{Ethical approval}

Ethical approval for the Healthy Thinking trial was obtained from the Human Research Ethics Committees of The Australian National University (2012/417) and the University of New South Wales (HC13117). All respondents were provided with full information about the trial before deciding whether to participate and gave online informed consent. Safety protocols were incorporated into the trial to ensure that participants who were distressed or actively suicidal could be readily linked to services (van Spijker et al., 2015).

\subsection{Analyses}

Difference in disability and HRQoL between participants with suicidal ideation only, participants with a single previous suicide attempt, and those with a history of multiple suicide attempts were assessed with Analysis of Variance (ANOVA). Tukey's post hoc test was used for pairwise comparisons ( $\alpha==0.05$ ). Exploratory backward stepwise multiple regression models were conducted to examine whether various demographic variables (age, gender, relationship status, education, employment status, and region) and clinical variables (depressive symptoms, anxiety, insomnia, alcohol consumption, suicidal ideation severity, and lifetime suicide attempt status) were associated with disability and HRQoL. Additional models that included the items that made up the total suicidal ideation severity score separately were run to examine associations of specific aspects of suicidal thoughts (such as frequency and controllability of thoughts) with disability and HRQoL. Dummy variables were created for categorical variables with more than two categories, using the largest category as the reference category. The model for each outcome (disability, MCS, and PCS) also included the two other outcomes as independent variables. Assumptions of normality and no multicollinearity were checked and found to be acceptable, with all Variance Inflation Factors (VIF) below 2.5. SPSS version 25 was used for all analyses.

\section{Results}

\subsection{Disability}

The mean disability score on the WHODAS for the overall sample was $19.2(S D==8.9$, range $0-43)$. Only eight participants $(1.9 \%)$ reported no difficulty in any activity (i.e., total disability score of 0 ). Three-quarters of the sample rated their overall health in the past month as moderate $(n=189,45.2 \%)$ or bad to very bad $(n=126$, $30.1 \%)$. Similarly, the majority of participants reported their difficulties interfered with their life moderately $(n=160,38.3 \%)$ or severely to extremely ( $n=156,37.3 \%)$. On average, difficulties were present 22 out of the 30 past days ( $S D==8.7$ ), which equates to $73.3 \%$ of the time. Participants were totally unable to carry out their usual activities 
Table 2

Predictors of disability in the full sample $(n=418)$.

\begin{tabular}{|c|c|c|c|c|c|c|c|c|}
\hline & $\begin{array}{l}\text { Full model } \\
B(S E)\end{array}$ & $\beta$ & $t$ & $p$ & $\begin{array}{l}\text { Best fitting mo } \\
B(S E)\end{array}$ & $\beta$ & $t$ & $p$ \\
\hline (Constant) & $6.39(5.17)$ & & 1.24 & 0.217 & $8.17(3.61)$ & & 2.27 & 0.024 \\
\hline Age & $0.11(0.03)$ & 0.15 & 3.39 & 0.001 & $0.09(0.03)$ & 0.12 & 3.07 & 0.002 \\
\hline \multicolumn{9}{|l|}{ Gender } \\
\hline Female & a & a & a & a & a & a & a & a \\
\hline Male & $-1.23(0.88)$ & -0.06 & -1.39 & 0.165 & b & b & b & b \\
\hline \multicolumn{9}{|l|}{ Relationship status } \\
\hline Single & a & a & a & a & a & a & a & a \\
\hline Married/de facto & $0.19(0.80)$ & 0.01 & 0.23 & 0.818 & b & b & b & b \\
\hline $\begin{array}{l}\text { Separated/divorced/widowed } \\
\text { Education }\end{array}$ & $-0.92(1.05)$ & -0.04 & -0.88 & 0.380 & $\mathrm{~b}$ & $\mathrm{~b}$ & b & $\mathrm{b}$ \\
\hline Associate degree or higher & a & a & a & a & a & a & a & a \\
\hline Trade or other certificate & $0.34(0.84)$ & 0.02 & 0.40 & 0.688 & b & $\mathrm{b}$ & b & $\mathrm{b}$ \\
\hline No post school qualification & $1.89(0.99)$ & 0.08 & 1.91 & 0.057 & $1.53(0.93)$ & 0.06 & 1.65 & 0.099 \\
\hline \multicolumn{9}{|l|}{ Employment status } \\
\hline Employed Full-time & a & a & a & a & a & a & a & a \\
\hline Employed Part-time & $0.60(0.94)$ & 0.03 & 0.64 & 0.526 & b & $\mathrm{b}$ & b & b \\
\hline Unemployed & $1.99(1.05)$ & 0.08 & 1.90 & 0.058 & $1.74(0.97)$ & 0.07 & 1.80 & 0.072 \\
\hline Not in labour force & $3.99(0.96)$ & 0.19 & 4.15 & $<0.001$ & $3.97(0.87)$ & 0.19 & 4.58 & $<0.001$ \\
\hline \multicolumn{9}{|l|}{ Region } \\
\hline Metropolitan & a & a & a & a & a & a & a & a \\
\hline Regional & $0.24(0.82)$ & 0.01 & 0.29 & 0.772 & b & $\mathrm{b}$ & b & $\mathrm{b}$ \\
\hline Rural/Remote & $2.36(1.05)$ & 0.09 & 2.24 & 0.026 & $2.34(1.01)$ & 0.09 & 2.33 & 0.020 \\
\hline \multicolumn{9}{|l|}{ Clinical characteristics } \\
\hline Depressive symptoms & $0.27(0.05)$ & 0.29 & 5.13 & $<0.001$ & $0.28(0.04)$ & 0.31 & 6.77 & $<0.001$ \\
\hline Anxiety symptoms & $0.03(0.08)$ & 0.02 & 0.32 & 0.747 & b & $\mathrm{b}$ & b & b \\
\hline Insomnia & $0.26(0.07)$ & 0.17 & 3.81 & $<0.001$ & $0.28(0.07)$ & 0.19 & 4.28 & $<0.001$ \\
\hline Alcohol Consumption & $-0.29(0.12)$ & -0.10 & -2.42 & 0.016 & $-0.34(0.12)$ & -0.11 & -2.91 & 0.004 \\
\hline C-SSRS ideation severity & $0.08(0.13)$ & 0.03 & 0.60 & 0.551 & b & $\mathrm{b}$ & b & \\
\hline \multicolumn{9}{|l|}{ Lifetime suicide attempt } \\
\hline Ideation only & a & a & a & a & a & a & a & a \\
\hline Single attempt & $0.96(0.92)$ & 0.04 & 1.04 & 0.298 & b & b & b & b \\
\hline Multiple attempts & $2.03(0.85)$ & 0.11 & 2.39 & 0.017 & $1.80(0.76)$ & 0.10 & 2.36 & 0.019 \\
\hline Disability (WHODAS-II) & c & c & c & & & & & \\
\hline SF-12 PCS & $-0.23(0.06)$ & -0.17 & -3.92 & $<0.001$ & $-0.24(0.06)$ & -0.17 & -4.12 & $<0.001$ \\
\hline SF-12 MCS & $-0.01(0.06)$ & -0.01 & -0.14 & 0.891 & b & $\mathrm{b}$ & & \\
\hline
\end{tabular}

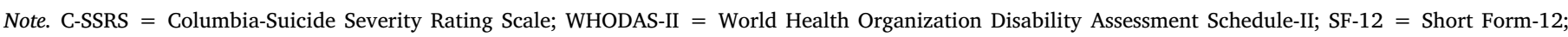
PCS = Physical Component Scale; MCS = Mental Component Scale.

${ }^{\text {a }}$ Reference category.

b Variable not included in best fit model.

c Variable not included in model as predictor variable.

or work for an average of 9 days $(S D==8.5)$ per month, and had to cut back or reduce their usual activities or work for an additional 15 days per month on average ( $S D==9.2$ ).

An ANOVA showed a significant difference in disability between groups according to lifetime suicide attempt status $\left(F_{2,415}=14.02\right.$, $p<0.001$ ). Tukey's post hoc test revealed that participants with multiple previous suicide attempts reported significantly higher overall disability $(22.2 \pm 8.9)$ compared to participants who never attempted suicide $(17.1 \pm 8.4, p<0.001)$ or attempted suicide once (18.9 $\pm 8.8, p=0.014)$. There was no difference between participants with suicidal ideation only and participants with a single previous suicide attempt ( $p=0.239$ ).

The multiple linear regression analysis (Table 2) showed that older age, not being in the labour force, and living in a rural or remote area were associated with disability. Higher levels of depression and insomnia, and lower levels of alcohol consumption and perceived physical health were also related to disability. In line with the ANOVA, multiple previous suicide attempts also had a significant association with disability. Adjusted $R^{2}$ was 0.399 for the full model and 0.405 for the model that best fit the data. Separate items regarding specific aspects of suicidal ideation (i.e., frequency and controllability of thoughts) were tested in the model but none of them added significantly to the variance explained by the model.

\subsection{Quality of life}

The mean PCS score for the overall sample was 40.7 ( $S D==6.5$, range 23.7-61.28) and the mean MCS score was $36.1(S D==5.9$, range 21.1-52.9). An ANOVA showed a significant difference in perceived physical health between groups according to lifetime suicide attempt status $\left(F_{2,415}=8.19, p<0.001\right)$. Participants with multiple previous suicide attempts reported significantly poorer perceived physical health $(38.9 \pm 6.3)$ compared to participants who never attempted suicide $(41.8 \pm 6.5, p<0.001)$ or attempted suicide once $(41.0 \pm 6.3, p=0.038)$. There was no difference in perceived physical health between participants with suicidal ideation only and participants with a single previous suicide attempt $(p=0.656)$. The ANOVA also showed no group differences for perceived mental health.

The multiple linear regression with perceived physical health as the dependent variable (see Table 3 ) showed that being separated, divorced or widowed, having vocational training, and living in a rural or remote area were associated with better perceived physical health. Clinical variables that were associated with worse perceived physical health were higher levels of depression, insomnia, and disability, and better perceived mental health. Consistent with the ANOVA, multiple previous suicide attempts also predicted worse perceived physical health. Adjusted $R^{2}$ was 0.220 for the full model and 0.230 for the model that fit these data best.

Worse perceived mental health was significantly predicted by not being in a relationship, higher levels of depressive symptoms, and 
Table 3

Predictors of PCS in the full sample $(n=418)$.

\begin{tabular}{|c|c|c|c|c|c|c|c|c|}
\hline & $\begin{array}{l}\text { Full model } \\
B(S E)\end{array}$ & $\beta$ & $t$ & $p$ & $\begin{array}{l}\text { Best fitting model } \\
B(S E)\end{array}$ & $\beta$ & $t$ & $p$ \\
\hline (Constant) & $55.57(3.23)$ & & 17.21 & $<0.001$ & $55.67(2.66)$ & & 20.93 & $<0.001$ \\
\hline Age & $0.01(0.03)$ & 0.01 & 0.19 & 0.843 & & $\mathrm{~b}$ & & \\
\hline \multicolumn{9}{|l|}{ Gender } \\
\hline Female & a & a & a & a & a & a & a & a \\
\hline Male & $0.42(0.73)$ & 0.03 & 0.57 & 0.566 & b & b & b & b \\
\hline \multicolumn{9}{|l|}{ Relationship status } \\
\hline Single & a & a & a & a & a & a & a & a \\
\hline Married/de facto & $0.80(0.66)$ & 0.06 & 1.21 & 0.228 & b & b & b & b \\
\hline $\begin{array}{l}\text { Separated/divorced/widowed } \\
\text { Education }\end{array}$ & $1.79(0.86)$ & 0.11 & 2.07 & 0.039 & $1.52(0.74)$ & 0.09 & 2.05 & 0.041 \\
\hline Associate degree or higher & a & a & a & a & a & a & a & a \\
\hline Trade or other certificate & $1.63(0.69)$ & 0.11 & 2.36 & 0.019 & $1.63(0.65)$ & 0.11 & 2.56 & 0.013 \\
\hline No post school qualification & $0.30(0.82)$ & 0.02 & 0.37 & 0.711 & & & & \\
\hline \multicolumn{9}{|l|}{ Employment status } \\
\hline Employed Full-time & a & a & a & a & a & a & a & a \\
\hline Employed Part-time & $0.25(0.78)$ & 0.02 & 0.32 & 0.751 & b & $\mathrm{b}$ & b & b \\
\hline Unemployed & $-0.15(0.87)$ & -0.01 & -0.18 & 0.779 & $\mathrm{~b}$ & $\mathrm{~b}$ & $\mathbf{b}$ & $\mathrm{b}$ \\
\hline Not in labour force & $0.13(0.81)$ & 0.01 & 0.16 & 0.872 & b & b & b & $\mathrm{b}$ \\
\hline \multicolumn{9}{|l|}{ Region } \\
\hline Metropolitan & a & a & a & a & a & a & a & a \\
\hline Regional & $0.62(0.68)$ & 0.04 & 0.91 & 0.362 & b & $\mathrm{b}$ & b & b \\
\hline Rural/Remote & $1.98(0.87)$ & 0.11 & 2.28 & 0.023 & $1.73(0.83)$ & 0.09 & 2.10 & 0.037 \\
\hline \multicolumn{9}{|l|}{ Clinical characteristics } \\
\hline Depressive symptoms & $-0.17(0.04)$ & -0.25 & -3.80 & $<0.001$ & $-0.17(0.04)$ & -0.25 & -4.43 & $<0.001$ \\
\hline Anxiety symptoms & $0.08(0.07)$ & 0.06 & 1.13 & 0.257 & b & $\mathrm{b}$ & b & \\
\hline Insomnia & $-0.12(0.06)$ & -0.11 & -2.07 & 0.039 & $-0.11(0.05)$ & -0.10 & -2.02 & 0.044 \\
\hline Alcohol Consumption & $0.06(0.10)$ & 0.03 & 0.56 & 0.576 & b & b & b & $\mathrm{b}$ \\
\hline C-SSRS ideation severity & $-0.11(0.10)$ & -0.05 & -1.07 & 0.287 & b & $\mathrm{b}$ & b & $\mathrm{b}$ \\
\hline \multicolumn{9}{|l|}{ Lifetime suicide attempt } \\
\hline Ideation only & a & a & a & a & a & a & a & a \\
\hline Single attempt & $0.09(0.76)$ & 0.01 & 0.12 & 0.901 & b & b & b & b \\
\hline Multiple attempts & $-1.21(0.70)$ & -0.09 & -1.72 & 0.086 & $-1.36(0.61)$ & -0.10 & -2.23 & 0.027 \\
\hline Disability (WHODAS-II) & $-0.16(0.04)$ & -0.22 & -3.90 & $<0.001$ & $-0.16(0.04)$ & -0.22 & -4.21 & $<0.001$ \\
\hline SF-12 PCS & c & c & c & c & c & c & & \\
\hline SF-12 MCS & $-0.12(0.05)$ & -0.11 & -2.28 & 0.023 & $-0.11(0.05)$ & -0.10 & -2.12 & 0.034 \\
\hline
\end{tabular}

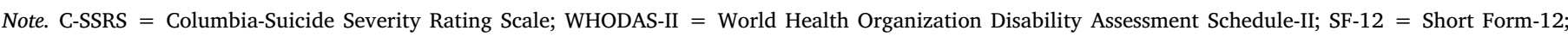
PCS = Physical Component Scale; MCS = Mental Component Scale.

a Reference category.

b Variable not included in best fit model.

c Variable not included in model as predictor variable.

higher levels of perceived physical health (see Table 4). Adjusted $R^{2}$ was 0.165 for the full model and 0.179 for the model that fit these data best.

As for disability, inclusion of separate items regarding specific aspects of suicidal ideation (i.e., frequency and controllability of thoughts) did not add significantly to the variance explained by either of the HRQoL models.

\section{Discussion}

This study reported on disability and HRQoL in a communitydwelling sample of adults who participated in an online suicide prevention trial. As expected, in the overall sample, disability was high and perceived mental and physical health was low, with particularly poor mental health as seen in previous research (Fairweather-Schmidt et al., 2016). Indeed, when compared to normative data for Australia (Andrews et al., 2009; Avery et al., 2004), the mean disability score on the WHODAS for the current sample (19.2) was significantly higher than the means of the general population $(3.10,95 \%$ confidence interval [CI] $[2.99,3.21])$, people with one mental disorder $(4.40,95 \%$ CI $[4.00,4.80])$, and people with more than one mental disorder $(8.70$, $95 \%$ CI $[8.13,9.27])$, which confirms the notion that suicidality is associated with severe disability. As noted by Andrews et al. (2009) people scoring between 10 and 48 on the 12-item WHODAS 2.0 are in the top $10 \%$ of the population distribution and likely to have clinically significant disability. Similarly, the mean PCS score on the SF-12 for our sample (40.7) was significantly lower than means of the general population $(48.90,95 \%$ CI $[48.60,49.40])$ and people with a mental health condition $(46.30,95 \%$ CI $[44.60,47.90])$. Furthermore, the mean MCS score on the SF-12 for our sample (36.1) was significantly lower than the mean of the general population $(52.40,95 \%$ CI $[52.00$, $52.80]$ ) and people with a mental health condition $(41.50,95 \% \mathrm{CI}$ $[39.80,43.00])$. The SF-12 results indicate suicidality is associated with substantially poorer perceived physical and mental health. Overall, these results are largely consistent with previous research indicating different forms of suicidality are associated with increased disability and decreased well-being (e.g., Lutz and Fiske, 2017; Sinclair et al., 2010).

The current study also showed that disability and perceived physical health was worst in those with a history of multiple suicide attempts, with no significant difference evident on these variables between those with no previous suicide attempt (ideation only) and those with only one previous suicide attempt. These findings were also consistent with our results from analyses taking into account other predictors of disability and perceived physical health, which indicated that multiple previous suicide attempts (but not single previous suicide attempts) was uniquely associated with higher disability and poorer perceived physical health. It may be the case that the decrements in physical health arise directly from injury stemming from more serious or repeat attempts, or that people with existing physical health conditions are more likely to report multiple attempts. Notably, there was no difference in perceived mental health across individuals with no previous suicide 
Table 4

Predictors of MCS in the full sample $(n=418)$.

\begin{tabular}{|c|c|c|c|c|c|c|c|c|}
\hline & $\begin{array}{l}\text { Full model } \\
B(S E)\end{array}$ & $\beta$ & $t$ & $p$ & $\begin{array}{l}\text { Best fitting model } \\
B(S E)\end{array}$ & $\beta$ & $t$ & $p$ \\
\hline (Constant) & $51.34(3.13)$ & & 16.41 & $<0.001$ & $50.15(2.53)$ & & 19.79 & $<0.001$ \\
\hline Age & $-0.10(0.03)$ & -0.01 & -0.19 & 0.847 & & b & b & b \\
\hline \multicolumn{9}{|l|}{ Gender } \\
\hline Female & a & a & a & a & a & a & a & a \\
\hline Male & $-0.31(0.69)$ & -0.02 & -0.45 & 0.651 & b & b & b & b \\
\hline \multicolumn{9}{|l|}{ Relationship status } \\
\hline Single & a & a & a & a & a & a & a & a \\
\hline Married/de facto & $1.40(0.62)$ & 0.12 & 2.24 & 0.026 & $1.37(0.59)$ & 0.11 & 2.34 & 0.020 \\
\hline Separated/divorced/widowed & $1.32(0.82)$ & 0.08 & 1.61 & 0.109 & $1.33(0.75)$ & 0.09 & 1.77 & 0.078 \\
\hline \multicolumn{9}{|l|}{ Education } \\
\hline Associate degree or higher & a & a & a & a & a & a & a & a \\
\hline Trade or other certificate & $0.39(0.66)$ & 0.03 & 0.60 & 0.550 & b & $\mathrm{b}$ & b & $\mathrm{b}$ \\
\hline No post school qualification & $-0.38(0.78)$ & -0.02 & -0.49 & 0.624 & b & b & b & b \\
\hline \multicolumn{9}{|l|}{ Employment status } \\
\hline Employed Full-time & a & a & a & a & a & a & a & a \\
\hline Employed Part-time & $-0.46(0.74)$ & -0.03 & -0.62 & 0.538 & b & b & b & b \\
\hline Unemployed & $0.91(0.82)$ & 0.06 & 1.11 & 0.267 & b & b & b & b \\
\hline Not in labour force & $-0.21(0.77)$ & -0.02 & -0.27 & 0.787 & b & b & b & b \\
\hline \multicolumn{9}{|l|}{ Region } \\
\hline Metropolitan & a & a & a & a & a & a & a & a \\
\hline Regional & $0.47(0.64)$ & 0.04 & 0.73 & 0.464 & b & $\mathrm{b}$ & b & $\mathrm{b}$ \\
\hline Rural/Remote & $1.10(0.83)$ & 0.06 & 1.32 & 0.188 & b & b & b & b \\
\hline \multicolumn{9}{|l|}{ Clinical characteristics } \\
\hline Depressive symptoms & $-0.28(0.04)$ & -0.45 & -6.86 & $<0.001$ & $-0.27(0.03)$ & -0.44 & -9.02 & $<0.001$ \\
\hline Anxiety symptoms & $0.02(0.07)$ & 0.02 & 0.36 & 0.720 & b & b & b & b \\
\hline Insomnia & $0.04(0.05)$ & 0.04 & 0.66 & 0.507 & b & b & b & $\mathrm{b}$ \\
\hline Alcohol Consumption & $0.05(0.10)$ & 0.02 & 0.50 & 0.620 & b & b & b & b \\
\hline C-SSRS ideation severity & $-0.11(0.10)$ & -0.06 & -1.12 & 0.266 & b & b & b & b \\
\hline \multicolumn{9}{|l|}{ Lifetime suicide attempt } \\
\hline Ideation only & a & a & a & a & a & a & a & a \\
\hline Single attempt & $0.63(0.72)$ & 0.04 & 0.87 & 0.383 & b & $\mathrm{b}$ & b & b \\
\hline Multiple attempts & $0.07(0.67)$ & 0.01 & 0.10 & 0.923 & b & $\mathrm{b}$ & b & $\mathrm{b}$ \\
\hline Disability (WHODAS-II) & $-0.01(0.04)$ & -0.01 & -0.14 & 0.891 & b & b & b & $\mathrm{b}$ \\
\hline SF-12 PCS & $-0.11(0.05)$ & -0.12 & -2.28 & 0.023 & $-0.10(0.04)$ & -0.12 & -2.24 & 0.026 \\
\hline SF-12 MCS & c & c & c & c & c & c & & \\
\hline
\end{tabular}

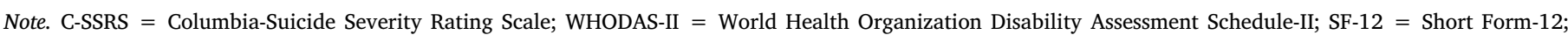
PCS = Physical Component Scale; MCS = Mental Component Scale.

a Reference category.

b Variable not included in best fit model.

c Variable not included in model as predictor variable.

attempt, a single previous attempt, and multiple previous attempts. Consistent with this, the analyses taking into account other predictors of perceived mental health did not show attempt status as a significant predictor. This finding suggests that many factors other than mental health status influence the transition from suicidal thoughts to attempts (Batterham et al., 2018; Fairweather et al., 2006; Klonsky et al., 2017). No other study has compared disability or HRQoL as a function of multiple attempts (Lutz and Fiske, 2017), so further exploration of these relationships is warranted. Finally, an unexpected result where worse perceived physical health had a small unique association with better perceived mental health emerged, after adjusting for other factors such as depression symptoms. Future research should examine whether this unexpected relationship can be replicated before attempting to provide a substantive interpretation, although the association between PCS and MCS has previously been found to be relatively weak (Gandek et al., 1998), and their associations with suicidal ideation may be complex (Fairweather-Schmidt et al., 2016).

One potential explanation for the association of more severe suicidality (i.e., multiple suicide attempts) with disability and poor perceived physical but not mental health may be a higher rate of physical health conditions in those participants reporting multiple suicide attempts. Physical health conditions are associated with an elevated risk of suicide independently of mental disorders (Ahmedani et al., 2017; Fairweather et al., 2006), and similarly, elevated risk of suicide is associated with physical health conditions independent of mental disorders (Goldman-Mellor et al., 2014). As such, having a physical health condition may impact on levels of disability and perceived physical health but not perceived mental health as the condition may not directly impact their mental health. Along similar lines, there may have been no difference between those with ideation only and those with only one previous suicide attempt because these groups had similar rates of physical conditions. Unfortunately, the current study did not collect data on participants' actual physical health conditions and thus was unable to explore this association further.

Another potential explanation is that suicide attempts can lead to long-term physical injuries (Borges et al., 2010; Li et al., 1997) and those with multiple previous suicide attempts may be more likely to have sustained such an injury due to the greater frequency of attempts. Such physical injuries may directly impact perceived disability and physical health while not necessarily lowering perceived mental health. As above, the lack of difference in disability and perceived physical health between those with no previous suicide attempt and those with only one previous suicide attempt may be attributed to both these groups having a lower likelihood of attempt-related physical injuries. Again, data was not collected regarding the presence of any attemptrelated physical injuries in participants, and future research should further examine this variable.

The current results highlight some important considerations. First, the significant disability and diminished quality of life associated with suicidality reinforces the continuing need for prevention and treatment. Indeed, this study suggests that early intervention is critical for individuals on the less severe end of the suicidality spectrum (i.e., 
ideation only, or single previous suicide attempt) to prevent the development of more severe forms of suicidality (i.e., multiple suicide attempts) and the associated high levels of reported disability and physical morbidity. In this regard, interventions that target suicidal thoughts, and interventions that aim to reduce future risk after a first suicide attempt (Shand et al., 2018) are integral. Second, our study suggests that individuals with multiple previous suicide attempts may have a higher likelihood of physical health conditions and/or attemptrelated physical injuries which are possible drivers of their higher disability and poorer perceived physical health. This raises the possibility that if interventions aiming to reduce risk in individuals with multiple previous suicide attempts can be tailored to take into account their physical morbidity, the interventions may more appropriately meet the needs of these individuals and potentially be more effective. For example, online interventions that target suicidal thoughts (e.g. van Spijker et al., 2018) could include content addressing the role of physical health conditions in the development of suicidal thinking and behaviour, and highlight the potential role of attempt-related physical injuries in perpetuating suicidal thinking.

Several limitations of the current study should be noted. First, participants in this study were recruited for a treatment trial, with a series of inclusion and exclusion criteria, and thus may not be representative of all people with suicidal thoughts and behaviours. For example, $77 \%$ of the sample identified as female, compared to $60 \%$ of individuals reporting ideation in the general population (Johnston et al., 2009). As such, future research with a broader sample of participants (e.g., suicide prevention service users) is recommended. Second, the number of past suicide attempts was assessed with a single self-report item and therefore reliant on people's own definition of a suicide attempt, which may not align with conventional research or clinical practice definitions. Third, the proportion of variance explained differed between the models for disability, perceived physical health, and perceived mental health, suggesting other variables not measured in this study may also be contributing to disability and perceived health. Finally, the current study utilised cross-sectional data and it is not possible to determine the direction of influence between the variables analysed.

Notwithstanding the limitations, this study found overall high levels of disability and low levels of perceived physical and mental health in a sample of individuals seeking online treatment for suicidal ideation. In particular, those with multiple previous suicide attempts had relatively higher disability and poorer perceived physical but not mental health when compared to the rest of the sample. As such, early intervention programs are needed to reduce the high levels of burden associated with suicidality, while interventions for individuals with multiple previous suicide attempts may benefit from increased attention to physical morbidity.

\section{Funding source}

This study was supported by a grant from the Australian National Health and Medical Research Council (NHMRC; GNT1046317) and forms part of the program of research conducted by the NHMRC Centre of Research Excellence in Suicide Prevention (GNT1042580). The funder did not have any role or ultimate authority in the study design; collection, management, analysis, and interpretation of data; writing of the paper; or the decision to submit the paper for publication. PB is supported by NHMRC Fellowship 1083311. AC is supported by NHMRC Fellowship 1122544. HC is supported by NHMRC Fellowship 1056964.

\section{Contributors}

BvS and HC conceptualized the Healthy Thinking study and oversaw trial implementation, data collection and interpretation. BvS, HC, PB, and AC contributed to study methods and obtained funding for the study. AWS contributed to the trial management and administration.
BvS conducted the statistical analyses, overseen by PB. BvS and QW cowrote the first draft of the manuscript and all other authors contributed to and reviewed the final manuscript.

\section{Declaration of Competing Interest}

BvS and HC are authors of the web-based self-help program mentioned in this manuscript. All other authors declare that they have no conflict of interest.

\section{Acknowledgements}

We sincerely thank the participants of the trial, as well as Daniela Solomon, Anna Frayne and Kale Dyer for their contributions to the trial management.

\section{Supplementary materials}

Supplementary material associated with this article can be found, in the online version, at doi:10.1016/j.jad.2019.11.050.

\section{References}

Ahmedani, B.K., Peterson, E.L., Hu, Y., Rossom, R.C., Lynch, F., Lu, C.Y., Waitzfelder, B.E., Owen-Smith, A.A., Hubley, S., Prabhakar, D., 2017. Major physical health conditions and risk of suicide. Am. J. Prev. Med. 53, 308-315.

Albrecht, G.L., Devlieger, P.J., 1999. The disability paradox: high quality of life against all odds. Social Sci. Med. 48, 977-988.

Andrews, G., Kemp, A., Sunderland, M., Von Korff, M., Ustun, T.B., 2009. Normative data for the 12 item WHO disability assessment schedule 2.0. PLoS ONE 4, e8343.

Avery, J., Dal Grande, E., Taylor, A., 2004. Quality of life in South Australia as measured by the SF12 health status questionnaire.

Batterham, P.J., Calear, A.L., Christensen, H., Carragher, N., Sunderland, M., 2018. Independent effects of mental disorders on suicidal behavior in the community. Suicide Life Threat. Behav. 48, 512-521.

Borges, G., Nock, M.K., Abad, J.M.H., Hwang, I., Sampson, N.A., Alonso, J., Andrade, L.H., Angermeyer, M.C., Beautrais, A., Bromet, E., 2010. Twelve month prevalence of and risk factors for suicide attempts in the WHO world mental health surveys. J. Clin. Psychiatry 71, 1617.

Bush, K., Kivlahan, D.R., McDonell, M.B., Fihn, S.D., Bradley, K.A., 1998. The AUDIT alcohol consumption questions (AUDIT-C). Arch. Intern. Med. 158, 1789-1795.

Christensen, H., Krysinska, K., Murray, S., 2015. The gap remains: NHMRC research funding for suicide and self-harm, 2000-2014. Med. J. Aust. 202, 525-526.

Cook, E.L., Harman, J.S., 2008. A comparison of health-related quality of life for individuals with mental health disorders and common chronic medical conditions. Public Health Rep. 123, 45-51.

Fairweather-Schmidt, A., Batterham, P., Butterworth, P., Nada-Raja, S., 2016. The impact of suicidality on health-related quality of life: a latent growth curve analysis of community-based data. J. Affect. Disord. 203, 14-21.

Fairweather, A.K., Anstey, K.J., Rodgers, B., Butterworth, P., 2006. Factors distinguishing suicide attempters from suicide ideators in a community sample: social issues and physical health problems. Psychol. Med. 36, 1235-1245.

G.B.D. DALYs, HALE Collaborators, 2017. Global, regional, and national disability-adjusted life-years (DALYs) for 333 diseases and injuries and healthy life expectancy (HALE) for 195 countries and territories, 1990-2016: a systematic analysis for the global burden of disease study 2016. Lancet 390, 1260-1344.

Gandek, B., Ware, J.E., Aaronson, N.K., Apolone, G., Bjorner, J.B., Brazier, J.E., Bullinger, M., Kaasa, S., Leplege, A., Prieto, L., Sullivan, M., 1998. Cross-validation of item selection and scoring for the SF-12 health survey in nine countries: results from the IQOLA Project. International Quality of Life Assessment. J. Clin. Epidemiol. 51, 1171-1178.

Goldman-Mellor, S.J., Caspi, A., Harrington, H., et al., 2014. . suicide attempt in young people: a signal for long-term health care and social needs. JAMA Psychiatry 71, 119-127.

Hoeymans, N., Schoemaker, C.G., 2010. The burden of disease of suicide and attempted suicide. RIVM Report 270342001/2010, in Dutch.

Jenkinson, C., Layte, R., Jenkinson, D., Lawrence, K., Petersen, S., Paice, C., Stradling, J., 1997. A shorter form health survey: can the SF-12 replicate results from the SF-36 in longitudinal studies? J. Public Health 19, 179-186.

Johnston, A.K., Pirkis, J.E., Burgess, P.M., 2009. Suicidal thoughts and behaviours among Australian adults: findings from the 2007 national survey of mental health and Wellbeing. Aust. N. Z. J. Psychiatry 43, 635-643.

Karimi, M., Brazier, J., 2016. Health, health-related quality of life, and quality of life: what is the difference? Pharmacoeconomics 34, 645-649.

Karimkhani, C., Trikha, R., Aksut, B., Jones, T., Boyers, L.N., Schlichte, M., Pederson, H., Okland, T., DiGuiseppi, C., Nasser, M., Naghavi, M., Vos, T., Yoong, S.L., Wolfenden, L., Murray, C.J., Dellavalle, R.P., 2016. Identifying gaps for research prioritisation: global burden of external causes of injury as reflected in the Cochrane database of systematic reviews. Injury 47, 1151-1157. 
Klonsky, E.D., Qiu, T., Saffer, B.Y., 2017. Recent advances in differentiating suicide attempters from suicide ideators. Curr. Opin. Psychiatry 30, 15-20.

Leonardi, M., Bickenbach, J., Ustun, T.B., Kostanjsek, N., Chatterji, S., 2006. The definition of disability: what is in a name? The Lancet 368, 1219-1221.

Leonardi, M., Raggi, A., Antozzi, C., Confalonieri, P., Maggi, L., Cornelio, F., Mantegazza, R., 2010. The relationship between health, disability and quality of life in myasthenia gravis: results from an Italian study. J. Neurol. 257, 98-102.

Li, G., Ling, J., DiScala, C., Nordenholz, K., Sterling, S., Baker, S.P., 1997. Characteristics and outcomes of self inflicted pediatric injuries: the role of method of suicide attempt. Injury Prevent. 3, 115-119.

Lutz, J., Fiske, A., 2017. Functional disability and suicidal behavior in middle-aged and older adults: a systematic critical review. J. Affect. Disord. 227, 260-271.

Morin, C.M., Belleville, G., Bélanger, L., Ivers, H., 2011. The insomnia severity index: psychometric indicators to detect insomnia cases and evaluate treatment response sleep34, 601-608.

Naghavi, M., 2019. Global, regional, and national burden of suicide mortality 1990 to 2016: systematic analysis for the global burden of disease study 2016. BMJ 364, I94.

Posner, K., Brown, G.K., Stanley, B., Brent, D.A., Yershova, K.V., Oquendo, M.A., Currier, G.W., Melvin, G.A., Greenhill, L., Shen, S., Mann, J.J., 2011. The columbia-suicide severity rating scale: initial validity and internal consistency findings from three multisite studies with adolescents and adults. Am. J. Psychiatry 168, 1266-1277.

Post, M., 2014. Definitions of quality of life: what has happened and how to move on. Top. Spinal Cord Inj Rehabil. 20, 167-180.

Sawyer Radloff, L., 1977. The CES-D scale: a self-report depression scale for research in the general population. Appl. Psychol. Meas. 1, 385-401.
Shand, F., Vogl, L., Robinson, J., 2018. Improving patient care after a suicide attempt. Aust. Psychiatry 26, 145-148.

Sinclair, J.M.A., Hawton, K., Gray, A., 2010. Six year follow-up of a clinical sample of selfharm patients. J. Affect. Disord. 121, 247-252.

Spitzer, R.L., Kroenke, K., Williams, J.B., Lowe, B., 2006. A brief measure for assessing generalized anxiety disorder: the GAD-7. Arch. Intern. Med. 166, 1092-1097.

Üstün, T.B., Chatterji, S., Kostansjsek, N., Rehm, J., Kennedy, C., Epping-Jordan, J., Saxena, S., von Korff, M., Pull, C., 2010. Developing the World Health Organization disability assessment schedule 2.0. Bull. World Health Organ. 88, 815-823.

van Spijker, B., Calear, A., Batterham, P., Mackinnon, A., Gosling, J., Kerkhof, A., Solomon, D., Christensen, H., 2015. Reducing suicidal thoughts in the Australian general population through web-based self-help: study protocol for a randomized controlled trial. Trials 16:62, 1-10.

van Spijker, B., van Straten, A., Kerkhof, A., Hoeymans, N., Smit, F., 2011. Disability weights for suicidal thoughts and non-fatal suicide attempts. J. Affect. Disord. 134, 341-347.

van Spijker, B., Werner-Seidler, A., Batterham, P., Mackinnon, A., Calear, A., Gosling, J., Reynolds, J., Kerkhof, A., Solomon, D., Shand, F., Christensen, H., 2018. Effectiveness of a web-based self-help program for suicidal thinking in an Australian community sample: randomized controlled trial. J. Med. Internet Res. 20, e15.

Ware, J., Kolinski, M., Keller, S., 1995. How to score the SF-12 physical and mental health summaries. A User's Manual. The Health Institute, New England Medical Centre, Boston, MA.

Ware, J., Kosinski, M., Keller, S., 1996. A 12-Item short-form health survey: construction of scales and preliminary tests of reliability and validity. Med. Care 34, 220-233. 